J. Dhaka National Med. Coll. Hos. 2011; 17 (01): 47-52

Review Article

\title{
Diagnosis and Treatment planning for Implant-Supported Fixed Prosthesis
}

\author{
Dr. Z.A. Shaheen ${ }^{1}$, Dr Z. Rehana ${ }^{2}$ \\ ${ }^{1}$ Associate Professor and Head, Department of Dentistry, Dhaka National Medical College, ${ }^{2}$ Dr. Zinat Rehena, Dental Surgeon, \\ Dhaka Dental College and Hospital.
}

\begin{abstract}
Implant prosthodontics were introduced in 1980s. Most unsuccessful cases are the result of a lack of communicating among the team members,insufficient knowledge of each members responsibilities, faulse assumptions etc. Understanding failures and their causes is more difficult with implant-supported prosthesis than with conventional prosthodontics. Careful diagnosis and treatment planning with a team approach prior to implant placement can help ensure a successful outcome. Diagnosis and treatment planning for use of a implant-supported fixed prosthesis are essential. The prognosis of the treatment plan should be confirmed by the implant team prior to treatment. All team members should understand the desired goals.
\end{abstract}

\section{Keywords: Implant,Prosthodontics,Prosthesis,Restoration,Appliance,Bone graft,Vertical dimension of occlusion(VDO).}

\section{Introduciton}

Implant reconstruction of the jaws is a prosthetically oriented discipline. The goal of prosthodotics is the maintainance and preservation of the remaining dentition and surrounding structures. Implant treatment can add greatly to patients perceptions of themselves and their surroundings. However fulfilling a patients desire for fixed restorations,rather than removable appliances,may not always be possible. An implant failure may occur after a less than optimum diagnosis and treatment plan by the implantologist

To avoid problems in implant dentistry,the clinicians should understand site and implant selection,optimum use of hard tissue grafting procedures, maintainance of soft tissues and papilla for aesthetics,replacement of one implant body for each missing tooth,and fit of the prosthesis.1-3. Ideally the practitioner evaluates the diagnosis and plans for the sequential treatment prior to any surgical consultation.

The diagnostic and treatment planning phase is critical in implant prosthodontics to avoid early and late failures. The proper sequencing of treatment should promote the maximum function during rehabilitation.

\section{Pretreatment Communications}

Communication between the clinician and the patient begins with their introduction each interviews and surveys the other. This first visit interview is important. Patients will describe their thoughts and attitudes about treatment,and they must be allowed to offer their comments. All patients have preconceptions of what their ideal treatment and result should be occur.Many times the patients preconceptions hinder optimum treatment planning and prognosis.

On the other hand patients confidence in the practitioner may be reinforced by a warm greeting an obvious interest in the patient and his or her problem, an attitude of concern for the patients welfare,and an unhurried atmosphere that affords the patient the opportunity to communicate comfortably with the doctor. Throughout this process, the implantologist evalutes the patient and the problems. The receptionist, chairside assistant,hygienist or dental technician should be welcomed and encouraged by the practitioner,as a part of a communication team, to aid in the appraisal of the patient.

The clinician must determine if he or she can satisfy this person both psychologically and dentally. The clinician must not commence any treatment if a favourable report does not exist.If there is an esthetic problem and the clinician does not think he or she can solve the patients cosmetic requirements satisfactorily,then treatment should not begin.

\section{Patient factors to consider}

Consider the following factors when determining whether or not a patient should be accepted for treatment.

\section{Adaptability}

This is an essential factor to consider because it may expedite the patients early acceptance of the prosthesis. Lack of adaptability may be revealed. Some patients may consider a prosthesis offensive to their body image,so acceptance may be fought and the prosthesis rejected.

\section{Motivation}

This should be strong general, and reinforced until the end of treatment. The process of adapting to the prosthesis may suffer for lack of motivation. Motivation of the patient is a vital component of successful prosthodontic service.

\section{Maintenance of adaptive capacity}

During treatment the patients emotional problems and conflicts should be identified and discussed. This may help prevent cancellation of scheduled appointments or postponement or abandonment of service. 
J. Dhaka National Med. Coll. Hos. 2011; 17 (01): 47-52

Willingness to participate in treatment.

Participation involves the patients acceptance of some responsibility.

Obsessions and compulsions.

Patient markedly fixed in habit patterns usually resistance to change.

Emotional stability : During the history taking,the clinician has an opportunity to encourage the patient to contribute towards the clinicians understanding of his or her physical and emotional

background and its relation to the planned dental service

Emotional stability

During the history taking, the clinician has an opportunity to encourage the patient to contribute towards the clinicians understanding of his or her physical and emotional background and its relation to the planned dental service.

\section{Medical history}

The medical history is an essential part of the diagnostic process and may have a direct bearing on the treatment plan and prognosis. 4 The medical history usually completed in the first visit, it becomes an important factor in establishing rapport and gaining confidence. Leading questions concerning past medical experience may reveal the patients level of pain tolerance, and further astute questioning will also reveal factors such as adaptability to change. The patient should be questioned about the following items:

\section{Cardiovascular problem \\ Neoplasms and treatment \\ Menopausal and post menopausal manifestation \\ Headaches}

Particularly severe migraine headaches, which may have a vascular, autonomic system back- ground in response to anxiety

\section{Insomnia, depression}

Gastrointestinal complaints including ulcer, colitis, nausea, vomiting, and chronic bowel problems. Allergies, asthma, or other similar disturbances that may have a psychological background. Medications being taken, including such details as length of time taken, dosage, frequency, and purpose ( sleep aid, muscle relaxation, hypertention, tranquilization, endocrine imbalance etc.)

\section{Immuno deficiencies such as HIV infection an AIDS}

Diabetes5- This diseases can involve loss of bone when the condition is uncontrolled or poorly controlled.

\section{Arteriosclerosis}

Any circulatory involvement may affect oral blood supply and limit healing capacities.

Renal diseases-Such problems may affect the patients water imbalance and therefore the stability of the prosthesis.

Endocrine imbalances- This can include menopausal changes and may influences emotional upsets as well as physical changes. Thyroid disease affect physical changes, especially in the bone of alveoiar ridge, as well as emotional upset.
Malnutritional effects-Dietary guidance may be necessary at all times to maintain health.

Sexually transmitted diseases-This diseases like syphilis, may involve the oral tissues and affect the central nervous system.

Diseases of the central nervous system.-Parkinsons and Bells palsy diseases affect the retention and control of the prosthesis

Medications.-Use of sedatives,sleeping pills,tranquilizer or muscle relaxants should alert the clinician to tactfully and sympathetically inquire as to the basis for such use.Cardiac drug may cause hyperplasia of the gum.

Smoking:-Contraindication for long term implant prognosis, especially with sinus grafting. 6-8

Age-Although this is not a specific contraindication,the amount and quality of the residual bone which can decrease over time, is the critical factor.

Acceptance-A patients unwillingness to accept loss of teeth whenthey are no longer serviceable will be a major hindrance to appropriate care.

Positive motivation-This may be determinant of successs.

Fear of dentistry.

The patient signature on a release form should be obtained if the clinician feels it necessary to contact his or her medical providers for further insight on any of the previously mentioned topics. Consultation with the physician is important in evaluating a chronically ill or mentally troubled patient and arriving at a diagnosis, treatment plan and prognosis.

\section{Diagnostic Evaluation}

This process begins with the introduction of the patient and continues with each visit. All diagnostic procedures and collected histories are aimed towards the goal of complete evaluation, so that a meaningful treatment plan may be determined. The following information should be considered in the diagnostic evaluation.

\section{Health information \\ Anatomical abnormalities}

This may include severly resorbed alveolar ridges, congenital def ormities like diminutive maxilla or mandible, undercut, tori, exoestosis, enlarge tongue and salivary gland. Age

Patient under 18 may not be candidates for implant replacement because their dental arches are not fully developed.

\section{Systemic illness}

These may reflected in poor tissue tone, low pain threshold, slow healing, sensivity to pressure, tissue fragility and xerostomia.

\section{Physical limitation}

Such limitation may result from accident, facial burns, malignancies or congenital defects.

\section{Occupation}


J. Dhaka National Med. Coll. Hos. 2011; 17 (01): 47-52 Social position may make specific demands on treatment objectives in relation to a patients occupation.

\section{Oral examination}

The oral examination is of utmost importance. It should include the following.

\section{Radiograph}

Dental radiograph and a panaromic radiograph should be taken to ascertain the condition of the bony structures and check the presence of pathological conditions like cyst,residual infection and diseases of bone. In addition CT scan might be indicated in implant restoration cases in which anatomic structures can not be evaluated by radiographs alone.

\section{Visual and digital examination}

A complete, meticulous visual and digital examination of the dental and oral structures should be completed and the clinician created a written record of the findings

\section{Impressions}

Maxillary and mandibular impressions should be made for construction of diagnostic cast. When mounted on an articulator permit evaluation of tissue contours in relation to space requirements, arch form, plane of occlusion and spacing of any remaining teeth that are to be retained and used in fixed, metal-acrylic resin first stage prosthesis.

\section{Vertical dimention of occlusion}

It should be established and verified, acceptable VDO is 3$4 \mathrm{~mm}$.

\section{Occlusal planes}

It should be determined, which may cause bending or breakage of the abutment or prosthetic retaining screws.

\section{Esthetic profile}

It should be created for the prospective patient, like lip contour, smile line, gingival line, incisal line, facial tooth contours, shade and shape etc.

\section{Previous treatment}

Problems with the previous prosthodontics should be evaluated. During the examination of existing prosthesis, the clinician must not show any reaction to previous treatment either verbally or visually.

\section{Treatment Planning and Methodology}

Fully extended complete-arch diagnostic casts of both maxilla and mandible are obtained from impressions. Two sets of impression are made and poured. The first set is kept on file, and the second set is used to duplicate mount, create a diagnostic waxup10 and perform an oral analysis. For partially edentulous situations these cast are fully extended to the anatomic limits of the edentulous area and provide an accurate representation of the remaining teeth. A prophylaxis is recommended if the patient has debris of any type on or around the remaining teeth. These casts should be mounted on a semiadjustable articulator with verified interocclusal records using a facebow transfer device.

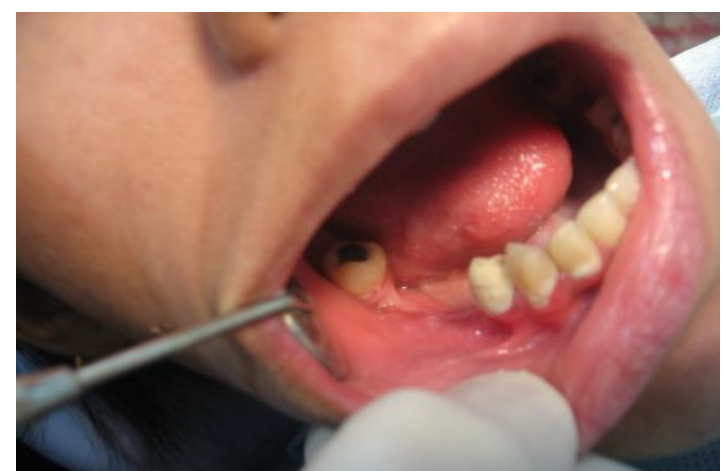

\section{Fig-1: Before treatment (Edentulous area)}

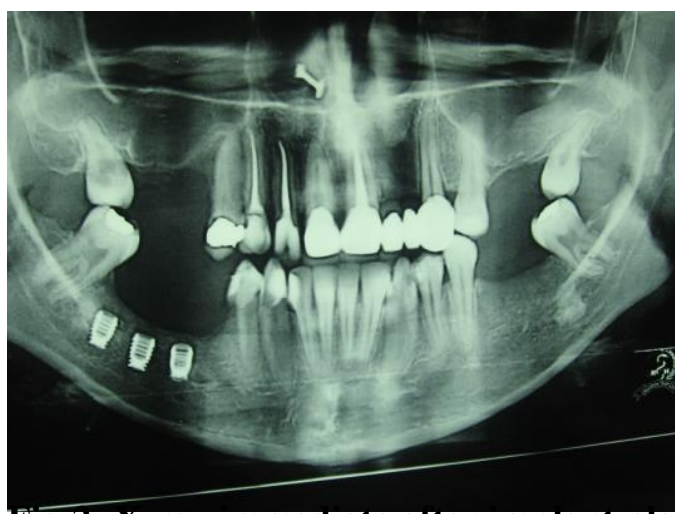

Fig-2: X-ray Immediate arter Implant placement.

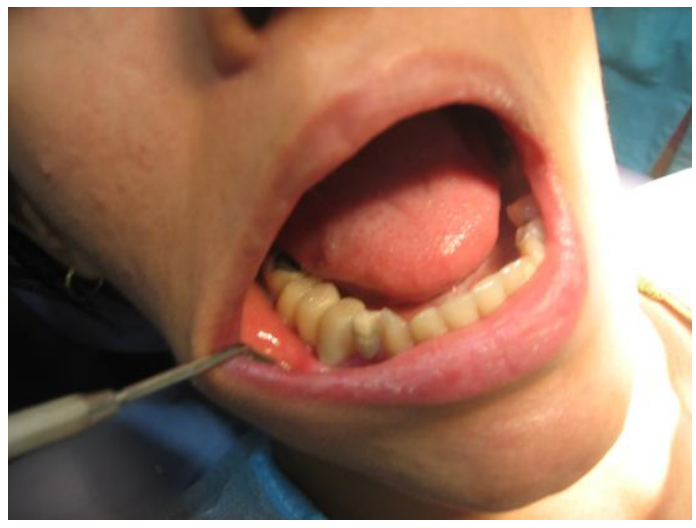

\section{Fig-3: After treatment (Implant supported prosthesis).}

The clinician should plan the prosthodontic treatment on the mounted diagnostic cast. The

occlusal plane should be established as of the occlusion in centric or eccentric relations, the incisal guidance for the fixed and removable implant-supported design, and the desired esthetic result. All this factors should be determined before beginning any irreversible or invasive dentistry in the patients mouth. Impression should be made of the completed diagnostic waxups to be used when fabricating the surgical templates 11 and the provisional and definitive prostheses. As mentioned previously the clinician should maintain one set of diagnostic casts. A second set should be sent to the surgeon and a third set sent to the laboratory technician with specific written instructions. These cast are also used as an aid in planning the sequence of treatment and are indication of the amount of grafting procedures needed for optimum esthetics, function, fabrication of surgical templates and provitional prostheses. They act as a template for the definitive prostheses. 
J. Dhaka National Med. Coll. Hos. 2011; 17 (01): 47-52 After making the appropriate casts, the clinician should then determine the sequence of treatment using all the acquired diagnostic aids. Every attempt should be made to avoid the completely edentulous state or the use of any remaining teeth to support a first stage provisional removable prosthesis $^{12}$.Use of first stage removable provisional appliances may cause implant loss due to the pressure on the surgical sites and micro-movement of the underlying implants. For the completely edentulous situations, whether in one or both arches, fully extended and adapted primary complete denture impressions are made boxed and poured. If there is any pathology beneath the existing complete denture, a provisional acrylic resin denture is fabricated, inserted and adjusted prior to a surgical consultation and implant placement. The patient is told not to wear the complete mandibular denture for at least 2 weeks following the surgical phase of implant placement. When a Caldwellluc approach to sinus lift has been used, the transitional RP is not worn for 3 weeks after surgery ${ }^{13}$. The clinician may use tissue conditioning materials inside the denture to relieve pressure on surgical sites and reduce the micromovement of recently placed implants after the delay period following implant placement. The tissue conditioning materials should be changed once a week until implant uncovering. consideration may be given to immediate loading $^{14,15}$ and transitional implants. ${ }^{16,17}$

In addition, prior to extraction of an anterior tooth with marked bone resorption, orthodontic treatment ${ }^{18}$ may be considered to extrude a tooth planned for extraction. This will bring the bone into a more favourable position incisally for enhanced cosmetics of the subsequent implant prosthesis. Once completed the surgeon will extract the tooth and place the implant according to the orientation established in a surgical template. This procedure may reduce the need for a bone graft at the time of implant placement.

Surgical considerations

During surgical consideration all the radiographs, a set of diagnostic casts, cast of completed diagnostic waxup, and intraoral radiographs should accompany the patient. The surgeon will determine the quantity and quality of bone and need for additional bone grafting. The length, width and type of implant are determined by the implantologist.

\section{Implant size}

The surgeon should place the longest and widest implant possible in each designed site. In full arch case one implant will be planed for each tooth to be replaced and occasionally two for every molar. This reduce the dending force of the underlying implants. When a singal molar is replaced by one implant screw loosing, bending,or breakage of the prosthetic or abutment screw may occur due to functional and parafunctional occlusal forces ${ }^{19,20}$. These problems are reduced by placing two implants ${ }^{21-24}$ if possible rather than only one ${ }^{4,5 .} 6 \mathrm{~mm}$ diameter implant, in a mesio-distal space of 10 to $14 \mathrm{~mm}$.

\section{Bone grafting}

Bone grafting used to create favourable implant-crown ratio,eliminate maxillary and mandibular cantilevers and avoid buccal offsets.

\section{Posterior implant placement}

In posterior partially edentulous areas creating a tripod effect with three posterior implants to avoid the rotation around the sagittal axis of the residual ridge that occurs when three posterior implants are placed in a straight line. A pontic between two implants should be avoided because the resultant mesiodistal bar will bend or flex under functional force. These force will bending movements on the implants and their components. This can lead to screw loosening or breakage of the implants due to increased stresses transmitted to the two implants. The tripod effect provides for axial loading and greatly reduces bending forces ${ }^{25}$. Thus it minimizes stress levels on implants and their components $^{26}$.

\section{Immediate implant placement}

After extraction immediate implant placement is preferred to delayed placement because the height of the alveolar bone is maintained. This is especially important in the anterior portions of the jaws because of the need for enhanced esthetics. For stabilized the immediate implant a longer implant can be used than would be selected if an extraction socket were allowed to heal before implant placement and the bone grafting is usually reduced . Immediate placement is not recommended, if there is infection around the apices of a tooth to be extracted or large amount of bone loss. In that case delayed placement and bone grafting are necessary.. To maintain a maximum amount of bone in a multi-rooted area the tooth is hemi- or trisected and each root is removed individually. Thus the interseptal bone may be maintained.

\section{Long axes}

Depending on the jaw relationship and bony contour, both the restorative and the surgeon should anticipate the long axes of the planned implants. This enables the team to select the desired abutments before second stage surgery and placed them at the uncovering surgery. Fabrication and insertion of a screw retained provisional prosthesis is then completed on the same day as abutment connection ${ }^{27,28}$.

\section{Esthetic profile}

Evaluation of the patients esthetic profile is required. The clinician should note the symmetry of the gingival margins, presence or absence of the interdental papillae, the smile line and the shape and shade of the maxillary anterior teeth. The relationship of the anterior teeth to the lip during function and the amount of rotation, overlapping and diastema should be evaluated. If the interdental papillae are present prior to treatment, should be maintained following implant procedures. Color photographs are used to record the initial appearance of these factors ${ }^{29,30}$.

Following fabrication of diagnostic casts, an incisal index is made. The incisal guidance would be changed if the vertical dimension of occlusion is decreased or increased. In the management of anterior implant prostheses, it is beneficial to use a surgical index coping transfer device to capture the position of the external hex of the implant during the first surgical visit,transfer it to a cast and fabricate a second stage screw-retained provisional prosthesis prior to the patients 
J. Dhaka National Med. Coll. Hos. 2011; 17 (01): 47-52 visit. The provisional prosthesis is prepared in the laboratory prior to the day of second surgery.

\section{Immediate loading}

The implantologist may consider the immediate loading of selected implants on the day of first surgery ${ }^{15,31}$ and more successful in mandible than in maxilla because of quality of bone. Immediate loading of implants is usually employed in the mandible when all remaining teeth or existing implants have failed and the patient can not function for any period of time without a prosthesis. The implants selected for loading must be stabilized in residual bone rather than a grafted site. When only two implants are loaded, due to anterior and posterior cantilevered movements, the torquing during normal function may cause implant failure. Another procedure in selected cases to eliminate a removable prosthesis and preserve function after implant placement is the use of a small-diameter provisional implant system. These are about $2 \mathrm{~mm}$ in diameter. The surgeon inserts the transitional implants alongside the primary selected implants during the same surgery. The clinician then fabricates a reinforced acrylic resin provisional prosthesis which is inserted on the same day as first-stage surgery ${ }^{14-17}$.

\section{Planning the Provisional prosthesis}

At the time of diagnosis and treatment planning clinician should determine the type of first stage provisional restoration to be employed. Transitional fixed first stage prosthesis is fabricated with a non-precious alloy substructure and acrylic resin veneer ${ }^{12}$. Three types of TRPD are available, all acrylic resin with buccal wrought clasp arms, acrylic resin with wrought gold wire clasp, and shoulder shallow occlusal rests and cast cobalt-chrome bases with acrylic resin denture teeth and bases, and eighter combination clasp or wrought gold,wrought wire clasp with shoulder shallow occlusal rest (long-term use) ${ }^{32}$. The first stage provisional restoration serve as template for the second stage provisional restoration and the definitive restoration. For full arch maxillary and mandibular implant supported provisional prosthesis the patients vertical dimension of occlusion should be maintained, centric relation and incisal guidance established. A progressive anterior group function from the central incisor to the first or second premolar is usually desirable ${ }^{29,23}$.

\section{Materials considerations for occlusal surfaces}

The patient should be informed of the materials employed on the occlusal surfaces and veneers. The rationale for use of the acrylic resin and the cast gold materials should be explained so the patient does not assume all veneering materials are porcelain. The use of an acrylic resin veneer, according to Shalak ${ }^{34}$ is more prudent-especially where the implants have been placed in sinus grafts or other types of bone grafts-as this will reduce forces on the underlying implants. To allow even wear between the natural teeth and implant sections, the same materials should be employed within the same arch and on opposing surfaces. Materials compatibility is needed for even occlusal wear and maintenance of the centric and eccentric occlusal contacts following completion of the prosthodontic treatment. Control of eccentric contacts is required. Occlusal forces should be directed to the long axis of the implants.

\section{Conclusion}

A complete patient evaluation and medical history is essential for obtaining the correct diagnosis determining the appropriate treatment plans and maximizing the chance for success. A diagnosis and prosthetic treatment plan should be based on accurate radiographs, analysis with a white waxup on mounted diagnostic casts, and an estimation of the time to be allotted for surgery, laboratory work and clinical treatment. It is readily apparent that actual clinical treatment is multifaceted. Time spent organizing the treatment plan is valuable and will be beneficial for patient understanding. The initial treatment outline may need alteration. In any event the patient must be flexible and understand the tentative nature of the initial treatment plan in order to avoid further problems.

\section{References}

1. Zinner et all ,solutions to complications in implant prosthodontics.1998;21:170-182.g

2. Naert IE. Patient evaluation and treatment planning.J.Dent1997; 25:S5-S11.

3. Eckert ES,Laney WR.Patient evaluation and Treatment planning for osseointregrated implants.1989; 33:599-618.

4. ElsubeihiES,ZarbGA. Implant prosthodontic in medical challenged patients.2002; 68:103-108.

5. Morris HF,et all implant survival in patients with type 2 diabetes 2000; 5;157-165.

6. Bain CA,Moly PK.The association between the failure of dental implants and cigarette smoking.1993; 8:609-615..

7. LambertPM,Moris HF.The influence of smoking 3 years clinical success of osseointegrated dental implants.2000; 5:79-89.

8. Schwartz-AradD, et all.Smoking and complications of endosseous dental implants. J periodontal2002; 73:153157.

9. Seide LJ.Adynamic Approcah to Restorative Dentistry.1980: chap1,15.

10. Culp L, Culp t. The art and science of diagnostic waxing.J Dent technol 2001;18(8):29-31.

11. BrielyTF.Surgical template fabrication.J Dent Technol 2000; 17(14):19-23.

12. Zinner ID,Panno FV,Pines MS.First stage fixed provisional restorations for implant rposthodontics.J Prostodont 1993;2(4):228-232.

13. Zinner ID,Small SA.Prosthetic management of the sinus graft case.1999; 157-165.

14. Schnitman PA,Wohrle PS.Ten-year results for Branemark implants immediately loaded with fixed prosthesis at implant placement. Int J Oral Maxillofac implants 1997; 2(4):495-503.

15. Gluser R,Ree A.Immediate occlusal loading of Branemark implants applied in various jaw bone regions:A prospective, 1 year clinical study.2001; 3:204213.

16. Babbush CA.Provisional implants.Implant Dent 2001; 10(2):113-119.

17. Froun $\mathrm{S}$, Emetiaz $\mathrm{S}$. The use of transitional implants for immediate fixed temporary prosthesis in cases of implant restorations.Pract Periodontics aesthet Dent1997; 10:737-746.

18. Salama H,Salama M.The orthodontic -periodontal connection in implant site development.Pract Periodontics Aesthet Dent1996; 8:923-932.

19. Ranger B,Jemt $T$.Forces and movements on Branemark implants.Int J Oral Maxillofac implants 1989; 4(3):241247. 
J. Dhaka National Med. Coll. Hos. 2011; 17 (01): 47-52

20. Parel SM,Sullivan DY. Esthetics and Osseointegration. 1989:36.

21. Balshi TJ. First molar replacement with an osseointegrated implants. 1990;21:61-65.

22. Baishi TJ,Harnandez RE.A comperative study of one imolant versus two replacing a single molar. Int $\mathrm{J}$ Oral Maxillofac implants 1996; 11:372-378.

23. Zinner ID,Small SA.Multiple implants for first molar prosthodontic.J Prosthodont 1996; 5:1548-165.

24. LeGall MG,Lauret JF. Mastication forces and implantbearing surface. Pract Periodontics Aesthet Dent 1994; 6:37-46.

25. Ranger B,Renoud F.Esthetic implant dentistry.2001:4767.

26. Renoud F,Ranger B.Factors in implant Dentistry.1999;39-66.

27. Zinner ID,Landa LS.Provisional screw retained fixed prostrhesis for implant Prosthodontics.1994;17:34-44.
28. Binon PP,Sullivan DY.Provisional fixed restorations technique for osseointegrated implants.J Calif Dent Assoc 1990; 18(1):23-30.

29. Schwartz H. Anterior guidance and esthetics in prosthodontics. Dent Clin North Am1987; 31:323-332.

30. Rifkin R. A comprehensive approach to treatment planning in esthetic Dentistry.Prac Periodontal Aesthet Dent 2000; 12:865-871.

31. Grunder U.Functional loading of immediate implants in edentulous area.Int J periodontics Restorative Dent 2001; 21:545-551

32. Zinner ID,Panno FV.Provisional prosthodontics appliances in the combination fixed removable reconstruction.Dent Clin North Am1989;33:379-397.

33. Schwartz H. Occlusal variations for reconstructing the natural dentition. J Prosthet Dent1986;55(1):101-105.

34. Skalak R.Osseointregration in Clinical Dentistry.1985:117-128.

Lazzara RJ.Surgical and Prosthetic considerations.Pract Periodontics Aesthet Dent 1994:6(9)55-62. 